OPEN ACCESS

Edited by:

Veronica Lazar

University of Bucharest, Romania

Reviewed by:

Fohad Mabood Husain,

King Saud University, Saudi Arabia

César de la Fuente,

Massachusetts Institute

of Technology, United States

*Correspondence:

Jintae Lee

jtlee@ynu.ac.kr

${ }^{\dagger}$ These authors have contributed equally to this work

Specialty section:

This article was submitted to Antimicrobials, Resistance

and Chemotherapy,

a section of the journal

Frontiers in Microbiology

Received: 26 November 2018 Accepted: 18 April 2019

Published: 08 May 2019

Citation:

Raorane CJ, Lee J-H, Kim Y-G, Rajasekharan SK, García-Contreras $R$ and Lee J (2019) Antibiofilm and Antivirulence Efficacies of Flavonoids and Curcumin Against

Acinetobacter baumannii.

Front. Microbiol. 10:990.

doi: 10.3389/fmicb.2019.00990

\section{Antibiofilm and Antivirulence Efficacies of Flavonoids and Curcumin Against Acinetobacter baumannii}

\author{
Chaitany Jayprakash Raorane ${ }^{1+}$, Jin-Hyung Lee ${ }^{1 t}$, Yong-Guy Kim ${ }^{1}$, \\ Satish Kumar Rajasekharan ${ }^{1}$, Rodolfo García-Contreras ${ }^{2}$ and Jintae Lee ${ }^{1 *}$ \\ ${ }^{1}$ School of Chemical Engineering, Yeungnam University, Gyeongsan, South Korea, ${ }^{2}$ Department of Microbiology \\ and Parasitology, Faculty of Medicine, National Autonomous University of Mexico, Mexico City, Mexico
}

Acinetobacter baumannii is well adapted to hospital environments, and the persistence of its chronic infections is mainly due to its ability to form biofilms resistant to conventional antibiotics and host immune systems. Hence, the inhibitions of biofilm formation and virulence characteristics provide other means of addressing infections. In this study, the antibiofilm activities of twelve flavonoids were initially investigated. Three most active flavonoids, namely, fisetin, phloretin, and curcumin, dose-dependently inhibited biofilm formation by a reference $A$. baumannii strain and by several clinical isolates, including four multidrug-resistant isolates. Furthermore, the antibiofilm activity of curcumin (the most active flavonoid) was greater than that of the well-known biofilm inhibitor gallium nitrate. Curcumin inhibited pellicle formation and the surface motility of A. baumannii. Interestingly, curcumin also showed antibiofilm activity against Candida albicans and mixed cultures of $C$. albicans and $A$. baumannii. In silico molecular docking of the biofilm response regulator BfmR showed that the binding efficacy of flavonoids with BfmR was correlated with antibiofilm efficacy. In addition, curcumin treatment diminished $A$. baumannii virulence in an in vivo Caenorhabditis elegans model without cytotoxicity. The study shows curcumin and other flavonoids have potential for controlling biofilm formation by and the virulence of $A$. baumannii.

Keywords: Acinetobacter baumannii, biofilm formation, curcumin, flavonoids, mixed biofilms, motility

\section{INTRODUCTION}

Most bacteria are able to form biofilms on various biotic and abiotic surfaces, and these films constitute structurally complex systems that defend microbial communities. Biofilm formation is a common cause of persistent infections by bacteria (Costerton et al., 1999), and resistance to eradication and high tolerance of conventional antimicrobial treatments are characteristic of bacterial biofilms (Wu et al., 2015; Lee et al., 2018).

Acinetobacter baumannii has been documented to be the most successful indigenous pathogen in healthcare institutions (Howard et al., 2012; Pakharukova et al., 2018). A. baumannii is an opportunistic Gram-negative bacillus that is responsible for a variety of nosocomial infections with high morbidity and mortality rates, these include, pneumonia, wound infections, bloodstream 
infections, urinary tract infections, and secondary meningitis (Howard et al., 2012; Liu et al., 2016). Furthermore, in intensive care neonatal and burns units, A. baumannii is one of the most commonly encountered pathogens (Seifert et al., 1994) (a claim shared with Pseudomonas aeruginosa and Staphylococcus aureus) (Paling et al., 2017a,b). Drug-resistant biofilm formation appears to play a vital role in the pathogenicity of A. baumannii (Qi et al., 2016), and biofilm development is critically dependent on the assembly of the $c s u A B A B C D E$ chaperon-usher, whereas pili production is required for adhesion to abiotic surfaces (Pakharukova et al., 2018). Furthermore, in A. baumannii it has been reported that biofilm formation and pili production were abolished by inactivation of the csuE gene (Tomaras et al., 2003), and that biofilm formation and motility are under the direct control of the two-component response regulator $\mathrm{BfmR}$, which acts as a master control switch for biofilm development (Russo et al., 2016).

Flavonoids are omnipresent in the plant kingdom and exhibit antioxidative, anti-inflammatory, anti-mutagenic, and anti-carcinogenic effects (Panche et al., 2016), that coupled with metal chelation and scavenge of free radicals (Abuelsaad et al., 2014). Recently, curcumin and several other flavonoids were reported to inhibit biofilm formation by Streptococcus mutans (Duarte et al., 2006), Aeromonas hydrophila (Abuelsaad et al., 2014), Candida albicans (Alalwan et al., 2017), S. aureus (Lee et al., 2012), and Escherichia coli O157:H7 (Lee et al., 2011) and persister cells formation in A. baumannii (Kaur et al., 2018). However, the antibiofilm activities of flavonoids have not been investigated against A. baumannii.

In this study, twelve flavonoids initially screened for nontoxic biofilm inhibitors against A. baumannii ATCC 17978, and the effects of three active biofilm inhibitors were further investigated with eight A. baumannii clinical isolates. In order to investigate the antibiofilm efficacy of the most active curcumin, confocal laser scanning microscopy (CLSM) and scanning electron microscopy (SEM) were utilized. Also, the effect of curcumin on pellicle formation and motility was studied. In addition, antibiofilm activity of curcumin was studied in two dual species biofilm models of $C$. albicans and A. baumannii. Furthermore, an in vivo Caenorhabditis elegans model was used to study the effect of curcumin on $A$. baumannii virulence.

\section{MATERIALS AND METHODS}

\section{Ethics Statement}

This study does not involve any human or animal participants nor does the study involve any invasion of privacy or accessing confidential information of individuals. The ethical committee of Yeungnam University has granted the exemption of ethical approval.

\section{Bacterial Strain and Chemicals}

A. baumannii ATCC 17978 and eight clinical A. baumannii isolates (ATCC BAA-1709, A 550, A 578, A 553, A 556, A 580, A 571, A 564) were obtained from burns patients at the National Rehabilitation Institute of Mexico; A. baumannii ATCC 17978 was used as a reference strain (Cruz-Muniz et al., 2017). For the dual biofilm experiment, we used $C$. albicans DAY185 (obtained from the Korean Culture Center of Microorganisms $^{1}$ ) and A. baumannii ATCC 17978. All experiments were conducted at $37^{\circ} \mathrm{C}$, and trypticase soy broth (TSB) and potato dextrose broth (PDB) media were used for the biofilm assay, Luria-Bertani (LB) medium for the pellicle assay, and motility agar (MA) medium in the motility experiment. Chemicals including twelve flavonoids viz. flavone (99\%), 6aminoflavone (97\%), 6-hydroxyflavone (98\%), apigenin (97\%), chrysin (97\%), curcumin (94\%), daidzein (98\%), fisetin (98\%), genistein (98\%), luteolin (98\%), phloretin (99\%), and quercetin (98\%), gallium nitrate (99.9\%), and crystal violet (90\%) were purchased from Sigma-Aldrich Co. (MO, United States). The structures of these flavonoids are provided in Figure 1A. TSB, PDB, LB media, and ethanol (95\%) were purchased from Becton Dickison and company (NJ, United States) and dimethyl sulfoxide (DMSO) from Duksan Pure Chemicals (Daegu, South Korea), respectively. All 12 flavonoids solutions were prepared by diluting them in DMSO that was also used as a negative control.

\section{Bacterial Culture and Minimum Inhibitory Concentration (MIC) Assay}

A. baumannii initially was streaked from $-80^{\circ} \mathrm{C}$ glycerol stock on trypticase soy agar (TSA) plates, and a single fresh colony was inoculated in TSB $(2 \mathrm{ml})$ in $14-\mathrm{ml}$ tubes and incubated at $37^{\circ} \mathrm{C}$ and $250 \mathrm{rpm}$ (Lee et al., 2012). Overnight cultures were reinoculated at 1:100 dilution in TSB. For cell growth measurements, a 1:100 inoculum was cultured in TSB $(25 \mathrm{ml})$ in $250 \mathrm{ml}$ flasks and incubated at $37^{\circ} \mathrm{C}$ overnight with agitation at $250 \mathrm{rpm}$. Optical densities were then measured at $600 \mathrm{~nm}$ using a spectrophotometer (Optizen 2120 UV, Mecasys, South Korea). Streaking and subculturing of C. albicans DAY185 was performed using potato dextrose agar (PDA) or PDB, unless otherwise specified (Manoharan et al., 2017). C. albicans was preserved at $-80^{\circ} \mathrm{C}$ in $1 \mathrm{ml}$ of $\mathrm{PDB}$ supplemented with $30 \%$ glycerol, and when needed, streaked on PDA plates. Plates were incubated for $48 \mathrm{~h}$ at $37^{\circ} \mathrm{C}$ and a fresh single colony was then inoculated into $25 \mathrm{ml}$ of PDB and cultured overnight at $37^{\circ} \mathrm{C}$. A minimum two independent cultures were used for each experiment.

MICs were determined (Betts and Wareham, 2014; Singh, 2014; Singh et al., 2017) using the National Committee for Clinical Laboratory Standards (2002) susceptibility testing guidelines in 96-well microtiter plates (SPL Life Sciences, Pocheon, South Korea). Briefly, an overnight culture at a dilution of 1:100 inoculated in TSB was cultured for $24 \mathrm{~h}$ in the presence of curcumin $(100,200$, or $500 \mu \mathrm{g} / \mathrm{ml})$ at $37^{\circ} \mathrm{C}$. After incubation cultures were spread on TSA plates, incubated for $24 \mathrm{~h}$ at $37^{\circ} \mathrm{C}$, and cell colonies were counted. Experiments were performed using at least two independent cultures.

\footnotetext{
${ }^{1}$ http://www.kccm.or.kr/
} 
A<smiles>Nc1ccc2oc(-c3ccccc3)cc(=O)c2c1</smiles><smiles>O=c1cc(-c2ccccc2)oc2ccc(O)c(OCCCCCCO)c12</smiles><smiles></smiles><smiles>O=c1c(-c2ccc(O)cc2)coc2cc(O)ccc12</smiles><smiles>CCOc1cc(O)cc2oc(-c3ccc(O)c(O)c3)cc(=O)c12</smiles>
Chrysin

Flavone Daidzein<smiles>COc1cc(/C=C/C(=O)CC(=O)/C=C/c2ccc(O)c(OC)c2)ccc1O</smiles>

B

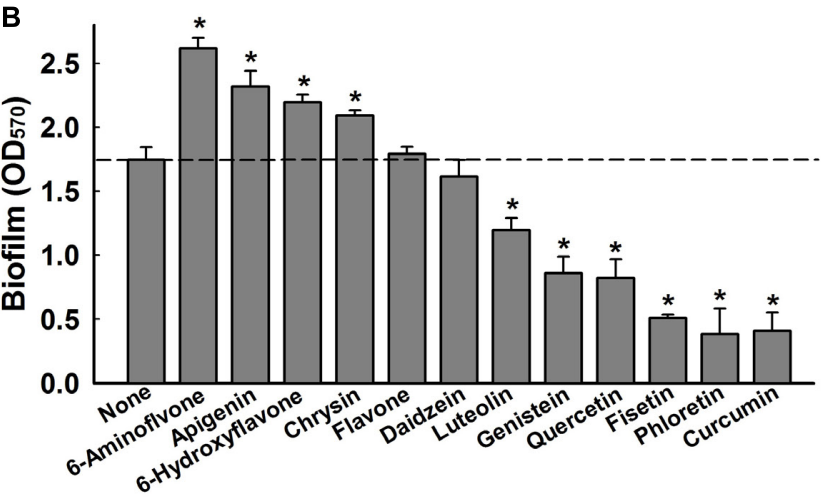

C

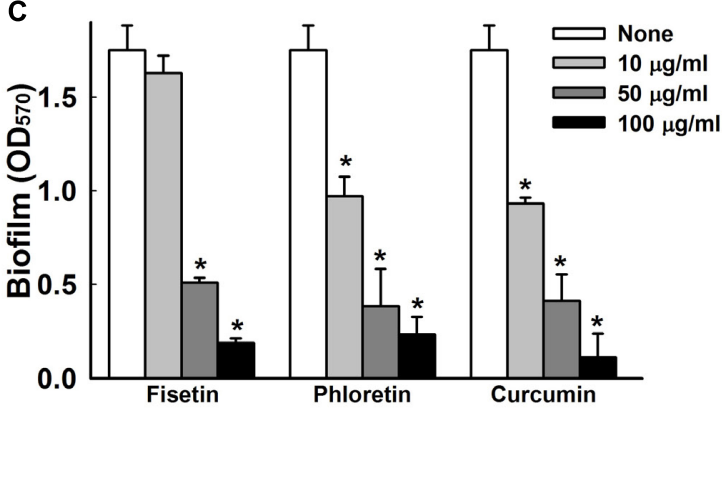

FIGURE 1 | Effects of flavonoids on A. baumannii biofilm formation. Chemical structures of the flavonoids used in this study (A). Effect of flavonoids on A. baumannil ATCC 17978 biofilm formation in TSB medium at $37^{\circ} \mathrm{C}$ after $24 \mathrm{~h}$ in 96 -well plates. Total biofilm formation $\left(\mathrm{OD}_{570}\right)$ in the presence of each flavonoid. All flavonoids were used at $50 \mu \mathrm{g} / \mathrm{ml}$, except luteolin, which was used at $25 \mu \mathrm{g} / \mathrm{ml}$ because of its antimicrobial activity (B). Dose-dependent effects of fisetin, phloretin, and curcumin on A. baumannii ATCC 17978 biofilm formation (C). ${ }^{*} p<0.05$ versus untreated controls.

\section{Crystal Violet Biofilm Assay and Antibiofilm Screening}

Static biofilm formation was assayed in 96-well polystyrene plates as previously reported (Lee et al., 2014). Briefly, cells in TSB (total volume $300 \mu \mathrm{l}$ ) inoculated at initial turbidity of 0.05 at $600 \mathrm{~nm}$ $\left(\mathrm{OD}_{600}\right)$ were cultured with or without for $24 \mathrm{~h}$ without shaking at $37^{\circ} \mathrm{C}$. The same amount of TSB was added to peripheral wells of 96-well plate to avoid edge effects. To quantify total biofilm formation, biofilms in 96-well plates were stained with $0.1 \%$ crystal violet for $20 \mathrm{~min}$, dissolved in 95\% ethanol, and absorbances were measured at $570 \mathrm{~nm}\left(\mathrm{OD}_{570}\right)$. Cell growth in 96-well plates was also measured at $620 \mathrm{~nm}\left(\mathrm{OD}_{620}\right)$. For initial anti-biofilm screening, we tested all twelve flavonoids at a concentration of $50 \mu \mathrm{g} / \mathrm{ml}$; results presented are averages of at least six replicate wells. Another static biofilm formation assay was performed in $14 \mathrm{ml}$ polyethylene tube, as previously described (Pour et al., 2011). A. baumannii ATCC 17978 cells were inoculated $1: 100$ in $5 \mathrm{ml}$ of TSB medium with curcumin at $0,10,20,50$, and $100 \mu \mathrm{g} / \mathrm{ml}$ and incubated for $24 \mathrm{~h}$ without shaking. Ring biofilms in $14 \mathrm{ml}$ polyethylene tubes were stained with crystal violet and results presented are the averages of at least three repetitions.

\section{Confocal Laser Scanning Microscopy}

Acinetobacter baumannii was inoculated at an $\mathrm{OD}_{600}$ of 0.05 in $3 \mathrm{ml}$ of TSB in glass bottomed confocal dishes (SPL life
Sciences, Pocheon, South Korea) for $24 \mathrm{~h}$ at $37^{\circ} \mathrm{C}$ with curcumin at 0,10 , and $50 \mu \mathrm{g} / \mathrm{ml}$ without shaking. To visualize biofilm structures, cells were stained with carboxyfluorescein diacetate succinimidyl ester (Invitrogen, Molecular Probes, Inc, Eugene, OR, United States). Biofilm structures were evaluated by CLSM (Nikon Eclipse Ti, Tokyo, Japan) (Lee et al., 2014), and their spatial characteristics were quantified using COMSTAT biofilm program $^{2}$ by analyzing at least four random positions in three independent cultures. To measure biofilm formation, color confocal images (20 image stacks) were converted to gray scale using ImageJ program ${ }^{3}$. COMSTAT biofilm software was used to determine biomasses $\left(\mu \mathrm{m}^{3}\right.$ per $\left.\mu \mathrm{m}^{2}\right)$, mean thicknesses $(\mu \mathrm{m})$, and substratum coverages (\%) (Runci et al., 2017).

\section{Assessment of Pellicle Formation}

A. baumannii can form pellicles more readily at air-liquid interfaces than other pathogenic Acinetobacter species (Chabane et al., 2014). The pellicle formation assay used was a modification of a previously described protocol (Marti et al., 2011). In brief, overnight bacterial cultures were diluted 1:100 in $5 \mathrm{ml}$ of LB broth and grown in glass tubes for $72 \mathrm{~h}$ at 25 and $37^{\circ} \mathrm{C}$ in the dark without agitation (Mussi et al., 2010). Amounts of pellicle material were assessed by adding $1 \mathrm{ml}$ of ethanol to tube underneath pellicle material, removing floating

\footnotetext{
${ }^{2}$ http://www.comstat.dk/

${ }^{3}$ https://imagej.nih.gov/ij/
} 
pellicles, and resuspending them in phosphate buffer saline (PBS) as previously reported (Giles et al., 2015). OD 600 values were measured using a spectrophotometer (Optizen $2120 \mathrm{UV}$, Mecasys, South Korea). Experiments were conducted in triplicate on three different days.

\section{Surface Motility Assay}

To assess surface motility with different concentrations of agar, MA containing $0.4 \%$ agarose, $1 \%$ tryptone, and $0.5 \%$ yeast extract was used (Clemmer et al., 2011), and MA medium supplemented with $0.25 \%$ agar (Eijkelkamp et al., 2011). Curcumin at 10 and $50 \mu \mathrm{g} / \mathrm{ml}$ concentration was added to MA, and DMSO (0.1\%) was used as a negative control. Overnight grown $\sim 0.2 \mu \mathrm{l}$ cultures of $A$. baumannii ATCC 17978 and three other multi-drug resistant clinical isolates (A 550, A 556, and A 580) were placed on motility plates using a sterile pipette tip. Sizes of halos produced by cells traveling across agar plates were measured after $9 \mathrm{~h}$ of incubation at $37^{\circ} \mathrm{C}$. Each experiment was performed using at least three independent cultures.

\section{Mixed Culture Biofilm Assay}

Because of its antibiofilm activity against C. albicans (Alalwan et al., 2017; Tan et al., 2018), we speculated curcumin would exhibit antibiofilm activity against a mixed culture of C. albicans and A. baumannii. Cells were inoculated together in PDB and TSB (50:50) mixed for C. albicans and A. baumannii at 1:50 ( $\mathrm{CFU} \sim 1 \times 10^{7}$ ) and 1:100 (CFU $\sim 7 \times 10^{7}$ ) dilution ratios from each with overnight cultures, respectively. Pure cultures of $C$. albicans and A. baumannii were tested at the same time. Biofilms in 96-well plates were stained with $0.1 \%$ crystal violet, dissolved $95 \%$ ethanol, and $\mathrm{OD}_{570}$ values were used to quantify total biofilm formation. Cell growth in 96-well plate were determined using $\mathrm{OD}_{620}$ values. Hyphal formation by $C$. albicans and mixed biofilm formation were assessed by SEM as previously described (Lee et al., 2014). Briefly, small pieces $(0.5 \mathrm{~cm} \times 0.5 \mathrm{~cm})$ of nylon filter were placed in wells of 96well plates containing $300 \mu \mathrm{l}$ cells/well. Cells were incubated in the absence or presence of curcumin at $37^{\circ} \mathrm{C}$ for $24 \mathrm{~h}$ without shaking. Prior to observation, biofilm samples were fixed with $2.5 \%$ glutaraldehyde and $2 \%$ formaldehyde for $24 \mathrm{~h}$, serially post fixed in PBS and osmium tetroxide, and dehydrated using an ethanol series (50, 70, 80, 90, 95, and 100\%) and isoamyl acetate. After critical-point drying, cells on filters were sputter-coated with palladium/gold and observed under an S4100 scanning electron microscope (Hitachi, Tokyo, Japan) at magnifications ranging from $\mathrm{x} 1,000$ to 10,000 using an accelerating voltage of $15 \mathrm{kV}$.

\section{Molecular Docking Simulations of Flavonoids With BfmR}

The molecular docking assays was conducted as previously described (Russo et al., 2016; Rajasekharan et al., 2017). Docking studies were performed to evaluate interactions between all twelve flavonoids and BfmR binding sites of $A$. baumannii (Protein Data Bank 6BR7). The three-dimensional structure of the beryllium fluorinated $\left(\mathrm{BeF}_{3}{ }^{-}\right)$receiver domain of A. baumannii BfmR resolved at $1.86 \AA$ was used for docking simulation. This $\mathrm{BfmR}$ domain consisted of two chains (A and B) and has a sequence length of 133 amino acids. For grid generation, beryllium fluorinated ligand was used as the centroid and ligands were docked at positions proximal to $\mathrm{BeF}_{3}-$ binding pockets (Draughn et al., 2018) using Schrodinger software 11.4 (Cambridge, MA, United States). A BfmR inhibitor 2-aminoimidazole and two biofilm inhibitors, virstatin and LED 209, were also docked at active sites. The glide score value and more specific target binding interactions to Asp15 and Asp58 were recorded.

\section{C. elegans Killing Assay}

The C. elegans killing assay used was a modification of a previously described protocol (Beceiro et al., 2014). Briefly, non-infected nematodes ( 20-30) [fer-15(b26);fem1(hc17)] were pipetted into 96-well plate containing M9 buffer and overnight curcumin $(50 \mu \mathrm{g} / \mathrm{ml})$ treated and untreated with A. baumannii and/or C. albicans cells. As a second dose curcumin was added to respective wells to make final concentration $50 \mu \mathrm{g} / \mathrm{ml}$ (total volume $300 \mu \mathrm{l}$ ). Nematodes were incubated at $25^{\circ} \mathrm{C}$ and viabilities were determined as previously described (Rajsekharan et al., 2018), by exposing them to LED or UV LED lights for 1030 s using an iRiS ${ }^{\mathrm{TM}}$ Digital Cell Imaging System (Logos BioSystems, South Korea). Three independent experiments ( $n=\sim 20-30$ ) were conducted.

\section{Statistical Analysis}

Replication numbers for assays are provided above and results are expressed as means \pm standard deviations. The statistical analysis was performed by one-way ANOVA followed by Dunnett's test using SPSS version 23 (SPSS Inc., Chicago, IL, United States). $P$ values of $<0.05$ were regarded significant and asterisks are used to indicate significant differences between treated and untreated samples.

\section{RESULTS}

\section{Impacts of Flavonoids on Biofilm Formation by $A$. baumannii}

The effects of the 12 flavonoids (Figure 1A) were initially investigated on A. baumannii ATCC 17978 biofilm formation in 96-well polystyrene plates using a crystal violet assay. Of the 12 flavonoids, luteolin, genistein, quercetin, fisetin, phloretin, and curcumin at $50 \mu \mathrm{g} / \mathrm{ml}$ exhibited biofilm inhibition, whereas 6-aminoflavone, apigenin, 6-hydroxyflavone, and chrysin increased biofilm formation, and the backbone flavone and daidzein had little effect (Figure 1B). Three flavonoids, that is, fisetin, phloretin, and curcumin, significantly and dose-dependently reduced biofilm formation, for example, these three flavonoids at 10 and $100 \mu \mathrm{g} / \mathrm{ml}$ reduced biofilm formation by $A$. baumannii ATCC 17978 by $>45$ and $>86 \%$, respectively (Figure 1C). 


\section{Antibiofilm Activities of Fisetin, Phloretin, and Curcumin Against $A$. baumannii Clinical Isolates}

Next, we investigated the antibiofilm activities of fisetin, phloretin, and curcumin against eight clinical A. baumannii strains (Cruz-Muniz et al., 2017). Of these strains, ATCC BAA1709, A 550, A 578, and A 553 were highly biofilm-forming, A 556, A 580, A 571 were intermediate, and A 564 had poor biofilm forming ability. Interestingly, fisetin, phloretin, and curcumin at $50 \mu \mathrm{g} / \mathrm{ml}$ all inhibited biofilm formation by the multidrug resistance (MDR) strains A 550, A 556, A 580, and A 564, and the antibiotic-sensitive ATCC BAA-1709 and A 571 strains. On the other hand, biofilm formation by two MDR strains, that is, A 553 (sensitive to colistin and amikacin), and A 578 (sensitive to colistin, imipenem, and meropenem) were not affected by fisetin, phloretin, or curcumin (Figure 2). Of these three flavonoids, curcumin was the most effective biofilm inhibitor against tested A. baumannii strains, and thus, it was the focus of subsequent studies conducted using the reference A. baumannii ATCC 17978 strain.

\section{Effects of Curcumin on Planktonic Cell Growth and Biofilm Formation}

The antibiofilm activity of curcumin was compared with that of gallium nitrate (a known biofilm inhibitor) (Runci et al., 2017). Both curcumin and gallium nitrate dose-dependently inhibited biofilm formation by the ATCC 17978 strain, though curcumin was superior to gallium nitrate at same concentrations (Figures 3A,B). For example, curcumin at 20 or $100 \mu \mathrm{g} / \mathrm{ml}$ decreased biofilm formation in polystyrene 96-well plate by 46 and $93 \%$, respectively, whereas gallium nitrate at these concentrations decreased biofilm formation by 24 and $67 \%$, respectively. Biofilm formation was also assessed in polyethylene tubes, as previously described (Pour et al., 2011), and curcumin was found to dose-dependently inhibit ring biofilm formation by $A$. baumannii (Figure 3D). We also examined the effect of curcumin on planktonic cell growth. The MICs of curcumin and gallium nitrate against ATCC 17978 strain were determined to be $>500$ and $>1000 \mu \mathrm{g} / \mathrm{ml}$, respectively. It was difficult to determine an exact MIC as curcumin precipitated from solution at higher concentrations, as previously reported (Betts and Wareham, 2014). Curcumin at concentrations up to $200 \mu \mathrm{g} / \mathrm{ml}$ slightly reduced (by $\leq 38 \%$ ) the planktonic cell growth of $A$. baumannii under shaking conditions in a flask (Figure 3C). These results indicated that antibiofilm activity of curcumin was not due to its antimicrobial activity, indicating curcumin may less prone to the development of drug resistance.

\section{Microscopic Observations of Biofilm Inhibition by Curcumin}

The antibiofilm effect of curcumin was further confirmed by CSLM and COMSTAT analysis. Interestingly, A. baumannii ATCC 17978 formed relatively thin surface biofilms on glass surfaces (Figure 3E) but robust ring biofilms on polyethylene (Figure 3D). Furthermore, the antibiofilm activity of curcumin was more marked on glass than polystyrene (Figure 3A) and polyethylene (Figure 3D) surfaces. For example, curcumin at $10 \mu \mathrm{g} / \mathrm{ml}$ markedly reduced surface biofilm formation on glass (Figure 3E), whereas at $100 \mu \mathrm{g} / \mathrm{ml}$ curcumin was more effective at preventing ring biofilm formation on polyethylene (Figure 3D). Biofilm reduction was also confirmed by COMSTAT analysis, which showed curcumin at 10 or $50 \mu \mathrm{g} / \mathrm{ml}$ significantly reduced biofilm biomasses, average thicknesses, and substrate coverage (Figure 3F). Specifically, biofilm biomass, thickness, and substrate coverage were reduced by curcumin at $10 \mu \mathrm{g} / \mathrm{ml}$ by $>95 \%$ versus untreated controls.

\section{Inhibitory Effect of Curcumin on Pellicle Formation}

A. baumannii colonizes the upper surfaces of static liquids and form biofilms at air-liquid interfaces by a process called pellicle formation, which is a type of biofilm formation (Kentache et al., 2017). The effect of curcumin on pellicle formation of ATCC 17978 and three other multi-drug resistant clinical isolates (A 550, A 556, and, A 580) were measured at 25

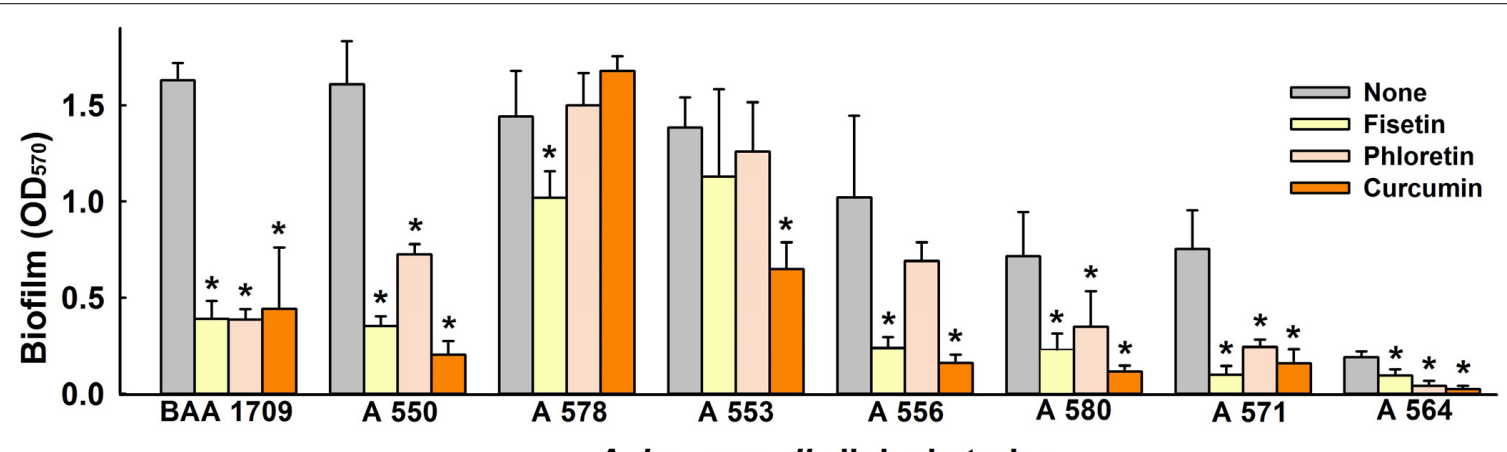

\section{A. baumannii clinical strains}

FIGURE 2 | Inhibitory effect of lead flavonoids on biofilm formation by A. baumannii clinical isolates. Biofilm formations by A. baumannii clinical isolates (ATCC BAA-1709, A 550, A 578, A 553, A 556, A 580, A 571, A 564) were quantified in the presence of fisetin, phloretin, or curcumin at $50 \mu \mathrm{g} / \mathrm{ml}$ after $24 \mathrm{~h}$ in $96-\mathrm{well}$ plates. $* p<0.05$ versus untreated controls. 

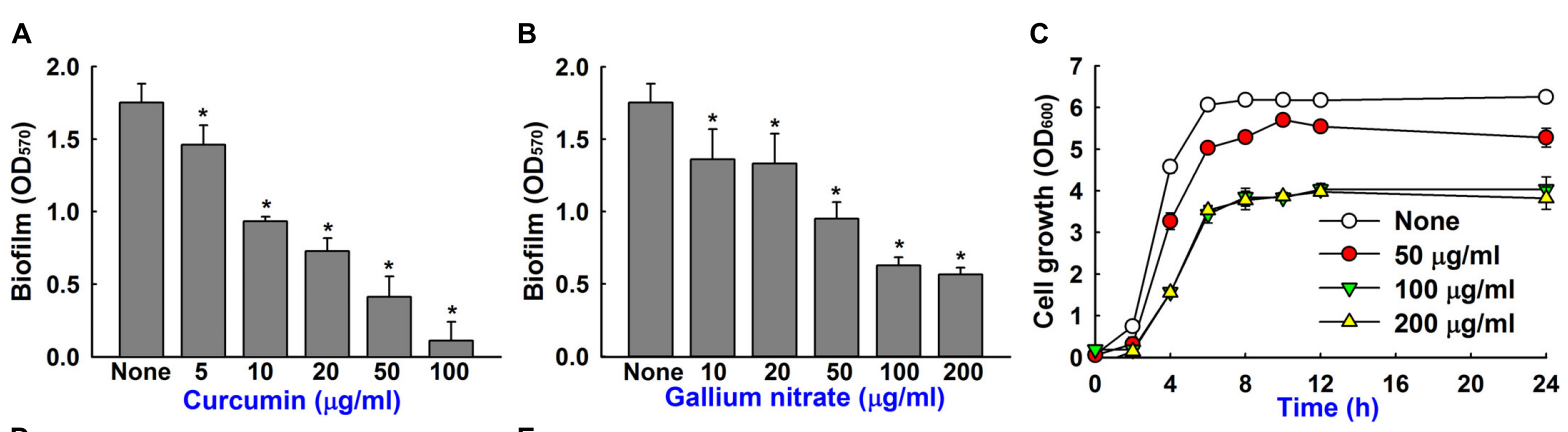

D
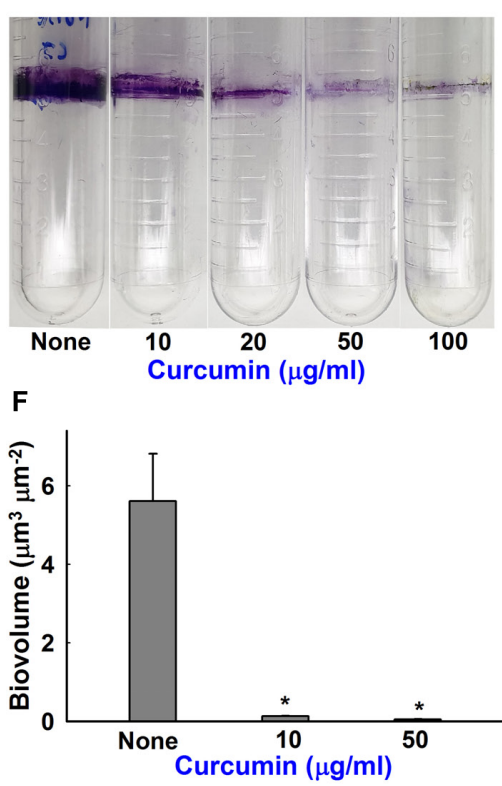

E
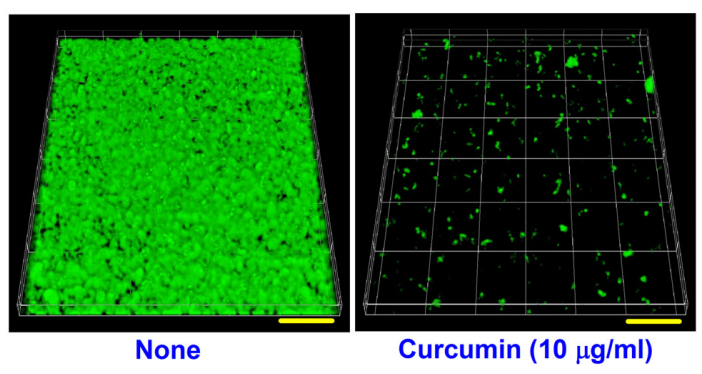

Curcumin $(10 \mu \mathrm{g} / \mathrm{ml})$

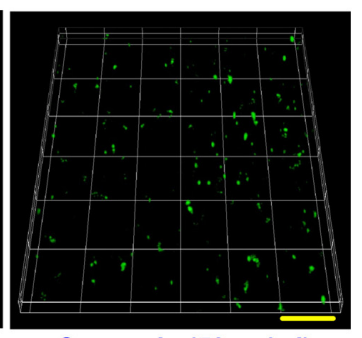

Curcumin $(50 \mu \mathrm{g} / \mathrm{ml})$
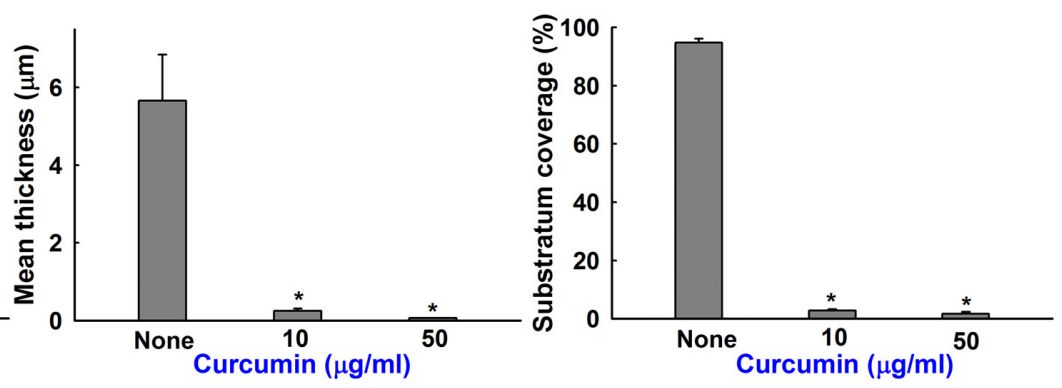

FIGURE 3 | Biofilm inhibition of A. baumannii by curcumin and gallium nitrate. Dose-dependent effects of curcumin on biofilm formation by A. baumannii ATCC 17978 (A). Effect of gallium nitrate on biofilm formation (B). Effect of curcumin on the cell growth of $A$. baumannii. Planktonic cell growth of $A$. baumannii was measured at $600 \mathrm{~nm}$ in $250 \mathrm{ml}$ flasks stirred at $250 \mathrm{rpm}$ (C). Dose-dependent effect of curcumin on A. baumannii ring biofilm formation on polyethylene when incubated at $37^{\circ} \mathrm{C}$ under static conditions (D). CLSM observation of biofilm inhibition by curcumin (E). Scale bar $=50 \mu \mathrm{m}$. Biofilm biomasses, mean thicknesses and substratum coverages spatial characteristics were quantified by COMSTAT analysis (F). * $p<0.05$ versus untreated controls.

and $37^{\circ} \mathrm{C}$ in $\mathrm{LB}$ medium without shaking. After $24 \mathrm{~h}$, a thin pellicle started to form at the liquid surface, and by the end of the third day, an opaque, solid pellicle covered the entire liquid surface (Figures 4A,B and Supplementary Figure S1D). Pellicle growth was found to be greater at $25^{\circ} \mathrm{C}$, which concurs with the results of Marti et al. (2011), and to be significantly inhibited by curcumin at $50 \mu \mathrm{g} / \mathrm{ml}$ (Figure 4C and Supplementary Figure S1F).

\section{Inhibition of Surface Motility by Curcumin}

Acinetobacter baumannii biofilm formation depends on the synthesis of pili, which are structures assembled by the $c s u A / B A B C D E$ chaperone-usher secretion system (Luo et al., 2015). Curcumin at $10 \mu \mathrm{g} / \mathrm{ml}$ reduced surface motility on $0.4 \%$ agarose and $0.25 \%$ agar (Figures 5A,B). Surface motility of ATCC 17978 with $0.4 \%$ agarose measuring mean halo diameters at $9 \mathrm{~h}$ were $1.4 \pm 0.6 \mathrm{~cm}$ for curcumin treatment and $5.6 \pm 1.6 \mathrm{~cm}$ for the non-treated control. Similarly, surface motilities measured in $0.25 \%$ agar using mean halo diameters were $0.3 \pm 0.2 \mathrm{~cm}$ for curcumin at $10 \mu \mathrm{g} / \mathrm{ml}$ and $6.6 \pm 2.3 \mathrm{~cm}$ for the control. Also, three clinical isolates (A 550, A 556, and A 580) were motile, which was significantly inhibited by curcumin (Supplementary Figures S1A-C,E). This is interesting since pili play a role both in biofilm formation and motility that were markedly abolished by curcumin.

\section{Mixed Culture Biofilm Inhibition by Curcumin}

Biofilm formation is a survival policy for bacteria and fungi in challenging environments (Wu et al., 2015). Crystal violet biofilm and SEM assays were used to examine the inhibitory effects of curcumin on mixed biofilms of $C$. albicans and $A$. baumannii. To form these dual biofilms, we used a (50:50) mixed medium of PDB and TSB to enable C. albicans and 

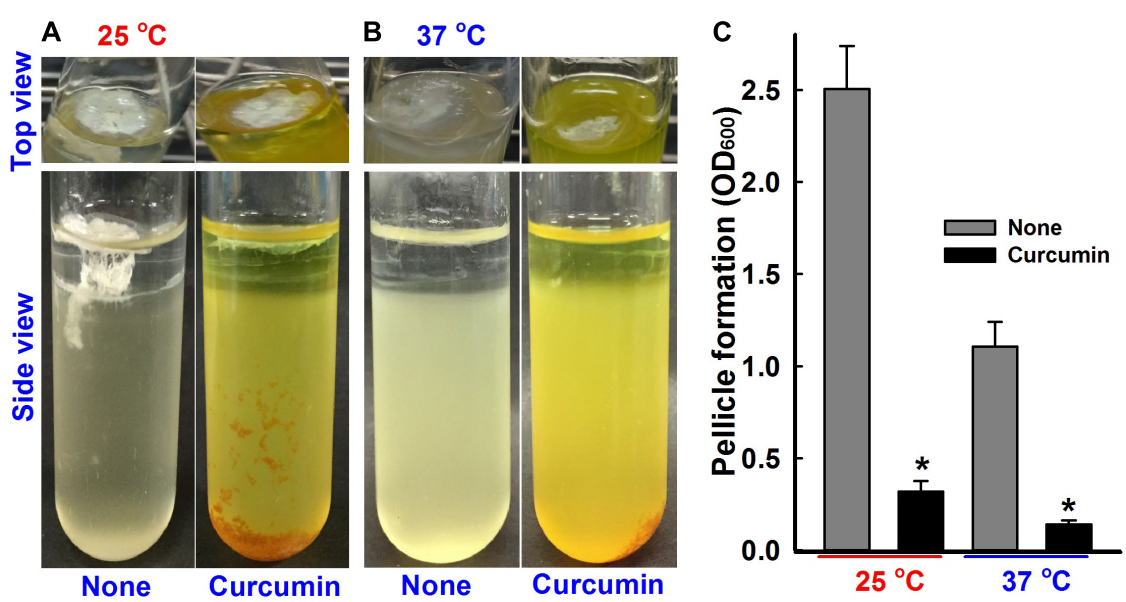

FIGURE 4 | Pellicle inhibition by curcumin. A. baumannii ATCC 17978 strain was grown in the presence or absence of curcumin $(50 \mu \mathrm{g} / \mathrm{ml})$ for $72 \mathrm{~h}$ at $25^{\circ} \mathrm{C}(\mathbf{A})$ or $37^{\circ} \mathrm{C}$ (B). Bar graphs represent pellicle formation as determined by spectrophotometry at $\mathrm{OD}_{600}$ (C). Experiments were performed using at least two independent cultures. ${ }^{*} p<0.05$ versus untreated controls.

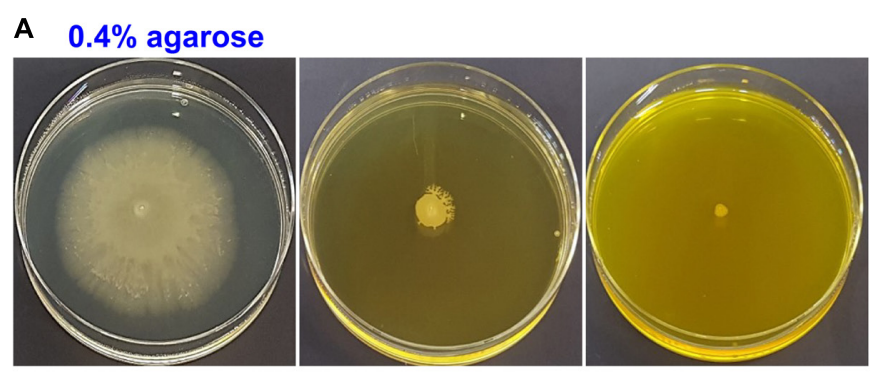

B $\quad 0.25 \%$ agar
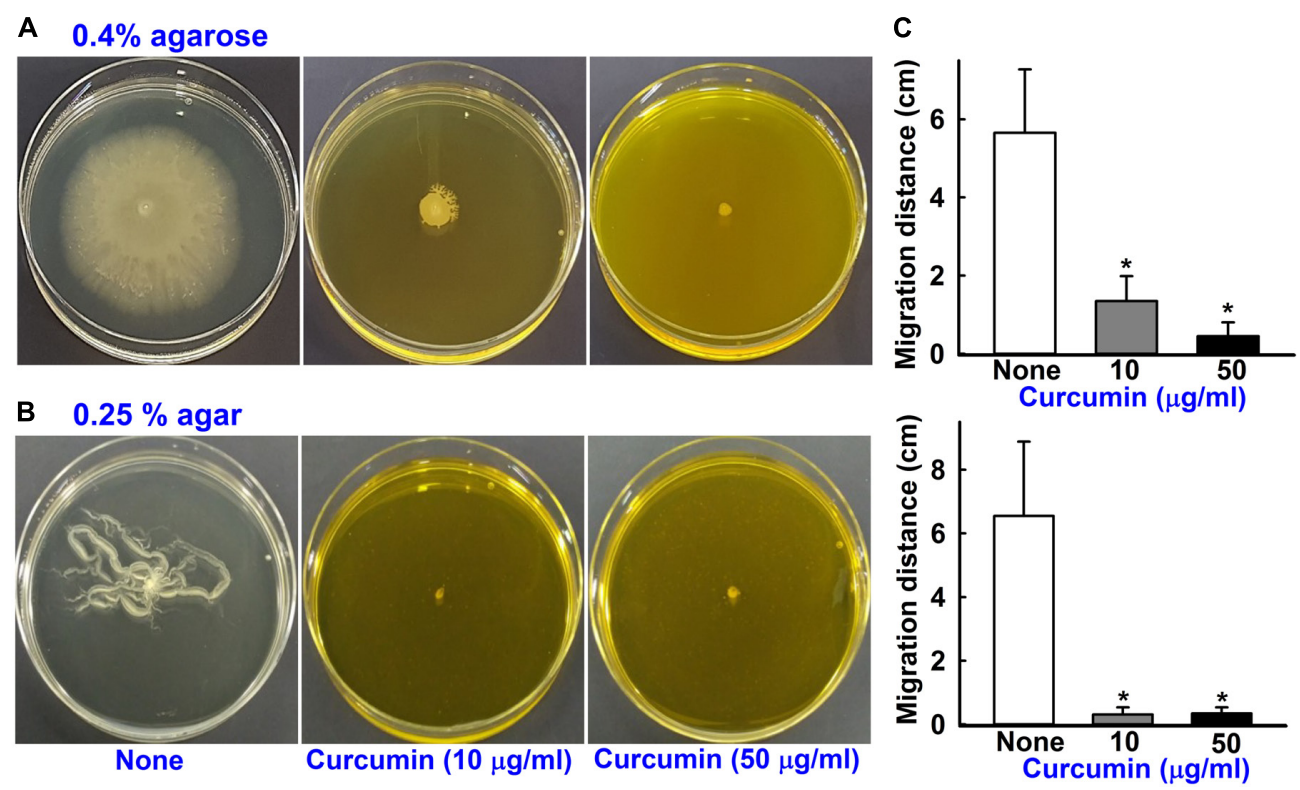

FIGURE 5 | Effects of curcumin on motility. The surface motilities on $0.4 \%$ agarose (A) and $0.25 \%$ agar (B) of $A$. baumannii ATCC 17978 were investigated after adding curcumin at 10 or $50 \mu \mathrm{g} / \mathrm{ml}$ to motility agar. The bar graphs represent swimming and swarming motility diameters in $\mathrm{cm}$ in the presence and absence of curcumin (C). ${ }^{*} p<0.05$ versus untreated controls.

A. baumannii growth, respectively. Under these conditions, decent biofilm formation $\left(1.0 \sim 3.0\right.$ at $\left.\mathrm{OD}_{570}\right)$ of individual C. albicans and A. baumannii strain and also co-culture of two species was observed (Figures 6A,B). As previously reported by Alalwan et al. (2017), curcumin dose-dependently inhibited biofilm formation by C. albicans (Figure 6A), for example, at $10 \mu \mathrm{g} / \mathrm{ml}$ curcumin reduced its biofilm formation by $>80 \%$. Importantly, curcumin at $20 \mu \mathrm{g} / \mathrm{ml}$ reduced mixed biofilm formation by $>85 \%$ (Figure 6B). SEM analysis showed that in mixed biofilms of $C$. albicans and $A$. baumannii, C. albicans formed large hyphae and few yeast cells, which were much larger than A. baumannii cells, and A. baumannii cells appeared to be encased in C. albicans hyphae (Figure 6C).

\section{Interactions of Flavonoids With the Biofilm Response Regulator BfmR}

Biofilm inhibition in A. baumannii is under the control of a $\mathrm{BfmR} / \mathrm{S}$, which is a two-component system (Liou et al., 2014). In this part of the study, we investigated interactions between several flavonoids and the $\mathrm{BeF}_{3}{ }^{-}$domain of $\mathrm{BfmR}$ (Figure 7 

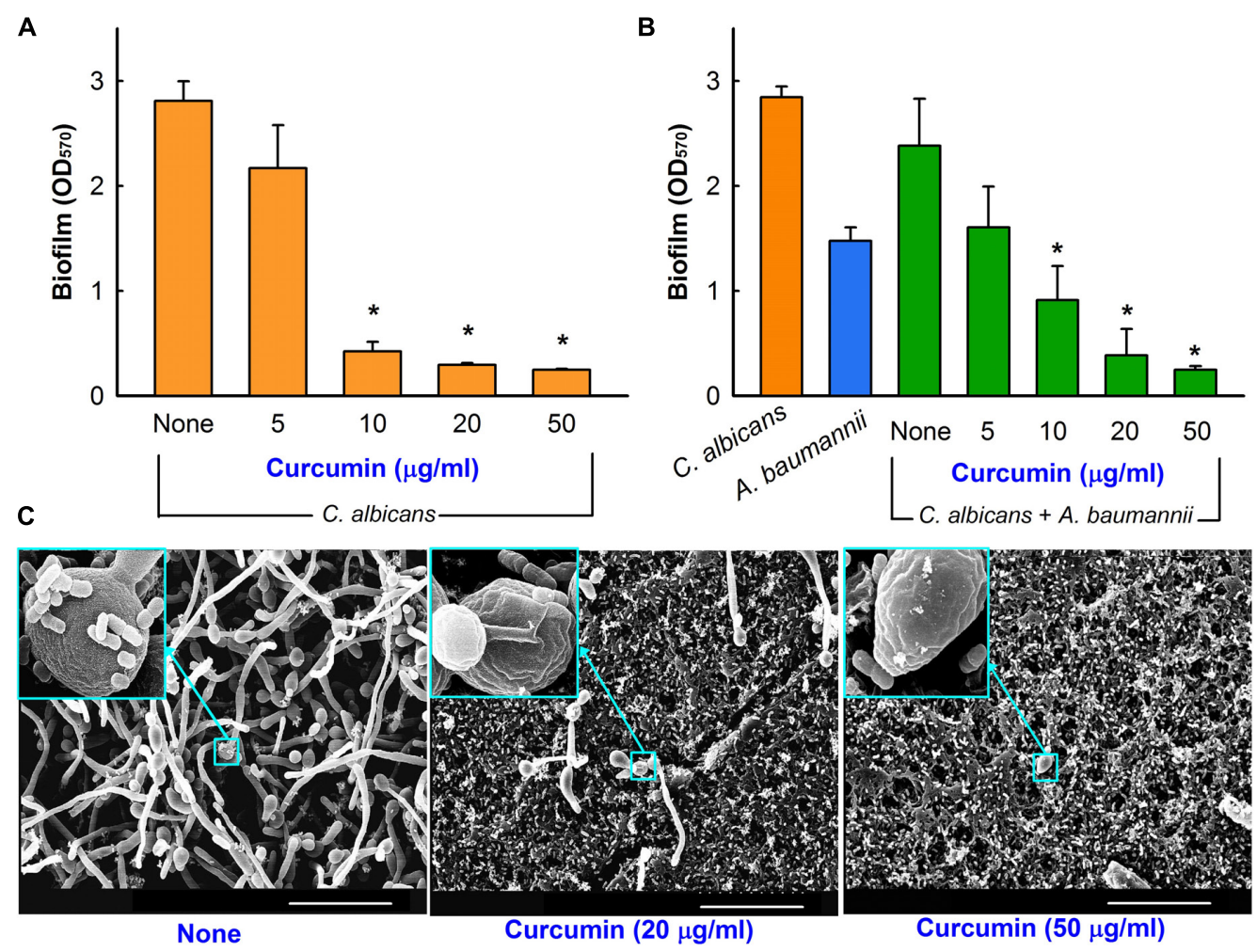

FIGURE 6 | Antibiofilm activity of curcumin in the mixed biofilm model. Antibiofilm effect of curcumin against C. albicans DAY185 in PDB medium (A). Antibiofilm effect of curcumin on mixed C. albicans DAY185 and A. baumannii ATCC 17978 biofilms was determined in a (50:50) mixed of PDB and TSB media after culture for $24 \mathrm{~h}$ in 96-well plates (B). SEM observation of $C$. albicans and A. baumannii mixed biofilms. In insets, the larger cells are $C$. albicans and the smaller cells are A. baumannii (C). Scale bar $=30 \mu \mathrm{m} .{ }^{*} p<0.05$ versus untreated controls.

and Supplementary Figure S2). The positive control, 2aminoimidazole (Thompson et al., 2012) exhibited hydrogen bond interactions with Asp15 and Asp58 and a Pi-Pi stacking with Lys107 (Figure 7A). Also, two known biofilm inhibitors (virstatin and LED209) interacted with Asp16 (adjacent to Asp15) and Lys107 (Figures 7B,C). Curcumin was found to interact well with the active site with a binding energy of $-38.7 \mathrm{kcal} / \mathrm{mol}$ (Supplementary Table S1). It formed backbone H-bonds with negatively charged Asp15 and two H-bonds with non-polar Val109 (Figure 7D). Fistein and quercetin also formed two $\mathrm{H}$-bonds with Asp15 and $\mathrm{Pi}-\mathrm{Pi}$ stacking with Lys107 (Figure 7F, Supplementary Figure S2, and Supplementary Table S1), while phloretin was found to interact better than fistein or quercetin with a binding energy of $-41.8 \mathrm{kcal} / \mathrm{mol}$, resulting from the formation of H-bonds with Asp15 and Asp58 and Pi-Pi stacking with Lys107 (Figure 7E, Supplementary Figure S2, and Supplementary Table S1). Several other flavonoids (6-aminoflavone, apigenin, 6-hydroxyflavone, luteolin, chrysin, flavone, and daidzein) were also tested which did not interact with the active site and showed poor interaction patterns (Supplementary Figure S2). Furthermore, our in vitro studies, showed these flavonoids did not inhibit biofilm formation (Figure 1B). Overall, BfmR binding efficacies of all 12 flavonoids were correlated with their antibiofilm efficacies. Based on in vitro and in silico findings, we speculate that the interaction of curcumin with $\mathrm{BfmR}$ could be one of the possible causes for its antibiofilm activity. However, further in vitro studies are required to confirm the curcumin/BfmR interaction.

\section{Curcumin Increased the Survival of C. elegans Exposed to A. baumannii}

Since A. baumannii and C. albicans kills the nematode C. elegans (Beceiro et al., 2014), a C. elegans killing assay was performed to examine the protective effect of curcumin. A. baumannii infection caused $80 \%$ C. elegans fatality (20\% survival) in 5 days (Figure 8A), but the presence of curcumin at $50 \mu \mathrm{g} / \mathrm{ml}$ reduced this to $35 \%$ (Figure 8A and Supplementary Figure S3), while the cell numbers of $A$. baumannii are similar (Supplementary Figure S4). Also, curcumin significantly attenuated the virulence of $C$. albicans in the nematode. These results show that curcumin effectively reduced the virulence of $A$. baumannii and C. albicans in our nematode model. Interestingly, mixed infection of $C$. albicans and $A$. baumannii showed much less virulence than the single pathogenic infection on $C$. elegans. This result confirms that $C$. albicans and A. baumannii are antagonistic each other and reduce their virulence against $C$. elegans as reported in references (Kostoulias et al., 2016). In addition, we investigated the chemical toxicity of curcumin against uninfected C. elegans. 
A

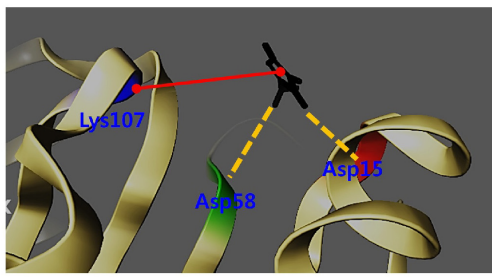

$A^{\prime}$

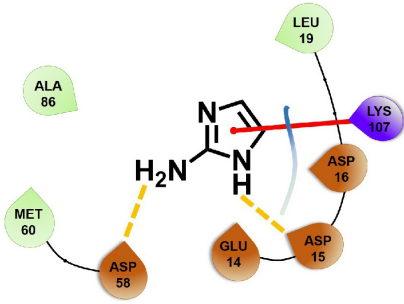

D

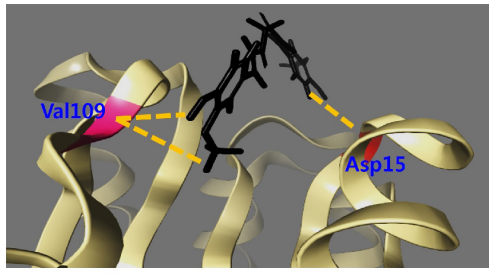

''
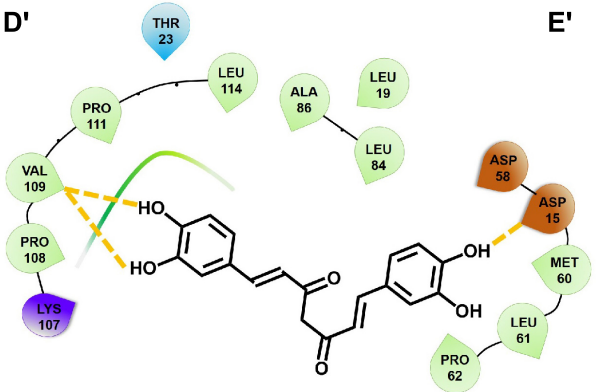

$E^{\prime}$
B

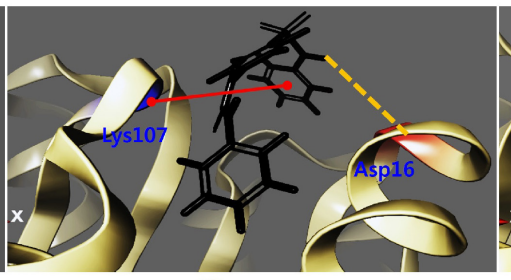

B'

C

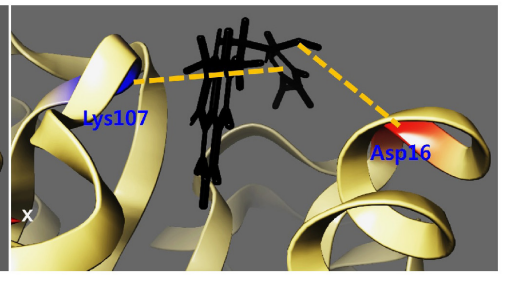

$C^{\prime}$

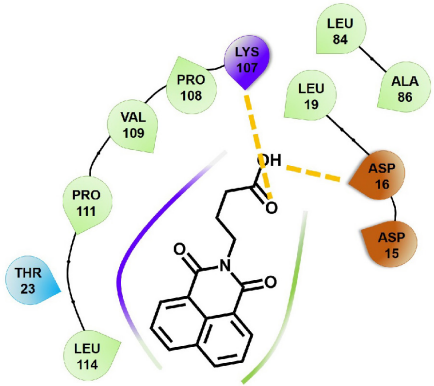

\section{F}
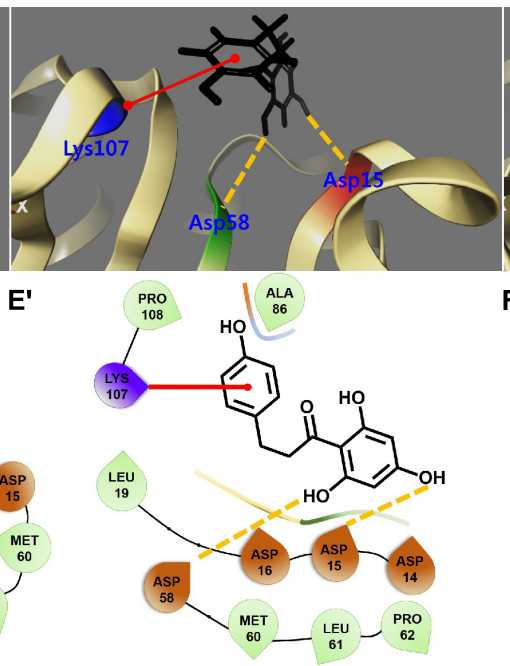

$F^{\prime}$
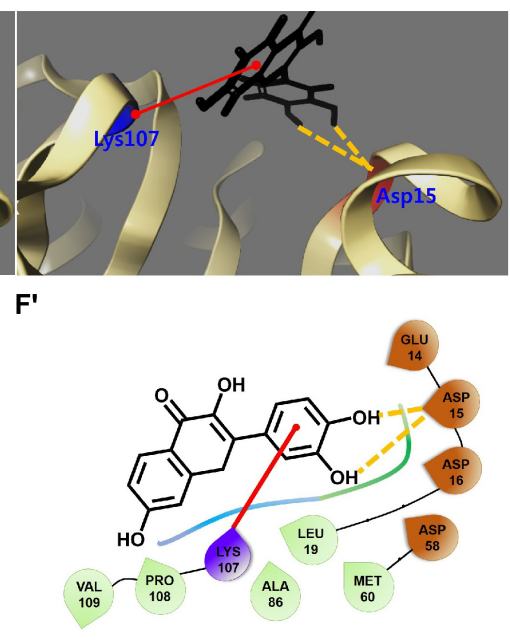

FIGURE 7| 2D and 3D interaction patterns of flavonoids with the N-terminal domain of BfmR. 3D binding orientations of 2-aminoimidazole (A), LED209 (B), virstatin (C), curcumin (D), phloretin (E), and fisetin (F) with respect to the active site of BfmR. The protein represented by ribbon. ( $\left.\mathbf{A}^{\prime}-\mathbf{F}^{\prime}\right)$ show $2 D$ interactions for respective ligands and surrounding amino acids residues. Negatively charged amino acids are depicted as red drops, hydrophobic amino acids as light green drops, and positively charged amino acids as violet drops. Backbone hydrogen bonds are shown as yellow dotted lines and Pi-Pi stacking is shown as red lines.

After 4 days trial, curcumin treated nematodes showed similar trends like the non-treated controls (Figure 8B), confirming that curcumin was nontoxic to worms, and did not affect the survival rate.

\section{DISCUSSION}

Flavonoids are a ubiquitous class of phytocompounds and considered prospective candidates for drug design (Merken and Beecher, 2000). Here, we report the biofilm inhibitory potentials of several flavonoids against the clinically relevant biofilm-forming bacterial pathogen A. baumannii. Of the twelve flavonoids tested, curcumin, fisetin, and phloretin most efficiently reduced biofilm formation by $A$. baumannii strains, including six clinical isolates (Figures 1, 2). In particular, curcumin inhibited ring biofilm formation, pellicle formation, motility, and mixed C. albicans and A. baumannii biofilm formation (Figures 3-6). Molecular docking analysis indicated flavonoids can interact with the biofilm response regulator BfmR (Figure 7 and Supplementary Figure S2).

Curcumin is an unstable, reactive, nonbioavailable compound (Nelson et al., 2017) while it is an established therapeutic 

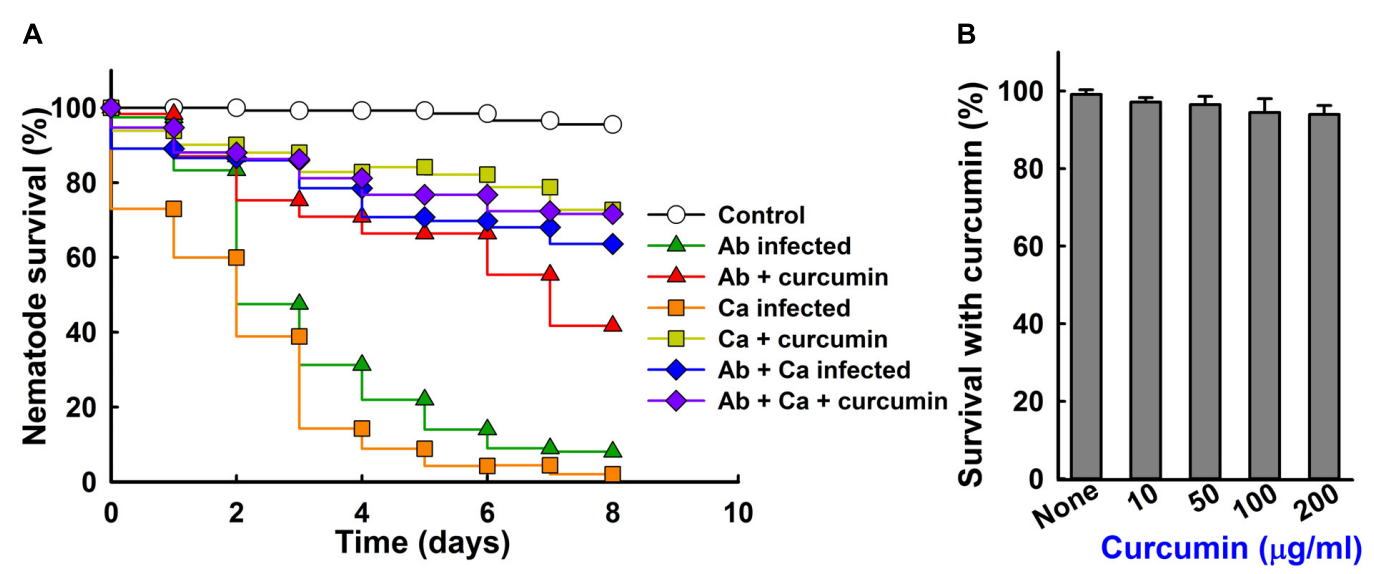

FIGURE 8 | Effect of curcumin on the survival of nematodes with $A$. baumannii and/or $C$. albicans infection. Liquid killing assay of $C$. elegans strain fer-15(b26);fem-1(hc17) infected with A. baumannii ATCC 17978 and/or C. albicans DAY185 in the presence of curcumin (A). Ab indicates A. baumannii ATCC 17978 and $\mathrm{Ca}$ indicates C. albicans DAY185. The effects of curcumin on non-infected nematodes after 4 days of exposure (B).

agent and is effective against various strains of Gram- negative and Gram-positive pathogens (Jaiswal and Mishra, 2018). Its mode of action at the molecular level has not been established, but it is thought to disrupt bacterial membranes (Tyagi et al., 2015; Teow et al., 2016). In the present study, we observed that at low concentrations, curcumin possibly inhibits biofilm formation by blocking BfmR, which is an interesting prospective therapeutic target in $A$. baumannii, as it has been shown inactivation of BfmR inhibits biofilm, motility and pellicle formation by A. baumannii (Russo et al., 2016). Recently, BfmR in A. baumannii was identified as a drug target, and as a result, several high-throughput molecular docking approaches have been used to identify small molecule BfmR inhibitors that interact strongly with biologically relevant sites in BfmR (Draughn et al., 2018). Similarly, we performed molecular docking to predict the binding efficacies of flavonoids with modeled BfmR. Active sites in BfmR were putatively identified by Draughn et al. (2018) who found several negatively charged amino acids in its active site. These included the conserved Asp58 and Asp15 residues coordinated with the $\mathrm{BeF}_{3}{ }^{-}$domain and a $\mathrm{Mg}^{2+}$ ion. In the present study, we found that our lead compounds (genestein, quercetin, fisetin, phloretin, and curcumin) and the standard inhibitors (virstatin, LED209, and 2-aminoimidazole) have strong positive interactions with BfmR. However, no such interactions were observed for seven other flavonoids (luteolin, apigenin, daidzein, chrysin, flavone, 6hydroxyflavone, and 6-aminoflavone), and these flavonoids did not exhibit antibiofilm activity (Figure 1B). Thus, our results suggest the inhibitory activities of flavonoids are related to BfmR binding modes.

In mixed culture of A. baumannii and C. albicans biofilms, the outer membrane protein of A. baumannii FhaB binds to Hyrlp (encoded by HYR1) of C. albicans, and it has been reported that HYR1 knockdown significantly reduces A. baumannii binding to C. albicans hyphae (Darwish Alipour Astaneh et al., 2017; Uppuluri et al., 2018). We observed mixed cultures C. albicans and A. baumannii formed substantial amounts of biofilm and that this was dose-dependently reduced by curcumin. We hope our findings will aid the fight against polymicrobial infections and believe they have significant disease management implications because they impact antimicrobial therapy selection against drug-resistant microorganisms. Furthermore, curcumin effectively reduced $A$. baumannii virulence in vivo in our $C$. elegans model without exhibiting toxicity (Figure 8). These findings show curcumin is a potential candidate for antivirulence strategies against persistent $A$. baumannii infections.

\section{CONCLUSION}

The expansion in drug resistance to conventional antibiotics has necessitated the developments of alternative antibiotic and antifungal agents. Over past decades, curcumin has been demonstrated to have potent antibiofilm activity and other pharmacological actions. Curcumin is marketed as a health supplement mainly for its antibacterial, antioxidant and antiinflammatory properties. However, the present study, curcumin was found to inhibit biofilm formation by $A$. baumannii strains and by $A$. baumannii and $C$. albicans mixtures and to attenuate $A$. baumannii virulence in our nematode model. These findings indicate curcumin has potential use as an alternative antibiotic or antifungal agent. However, we recommend more investigations be conducted to better understand the broad action of curcumin before efforts are made to develop antibiofilm or antivirulence agents based on curcumin.

\section{AUTHOR CONTRIBUTIONS}

$\mathrm{CR}, \mathrm{J}-\mathrm{HL}$, and Y-GK performed in vitro experiments, and analyzed the data. SR performed the docking studies. RG-C 
provided bacterial isolates and helped to design study. CR, J-HL, SR, and JL designed the study and wrote the manuscript. All the authors read and approved the final manuscript.

\section{FUNDING}

This research was supported by the Basic Science Research Program through the NRF funded by the Ministry of Education (2018R1D1A3B07040699 to J-HL and 2018R1D1A1B07044288

\section{REFERENCES}

Abuelsaad, A. S., Allam, G., and Al-Solumani, A. A. (2014). Hesperidin inhibits inflammatory response induced by Aeromonas hydrophila infection and alters $\mathrm{CD}^{+} / \mathrm{CD}^{+} \mathrm{T}$ cell ratio. Med. Inflamm. 2014:393217. doi: 10.1155/2014/ 393217

Alalwan, H., Rajendran, R., Lappin, D. F., Combet, E., Shahzad, M., Robertson, D., et al. (2017). The anti-adhesive effect of curcumin on Candida albicans biofilms on denture materials. Front. Microbiol. 8:659. doi: 10.3389/fmicb.2017.00659

Beceiro, A., Moreno, A., Fernandez, N., Vallejo, J. A., Aranda, J., Adler, B., et al. (2014). Biological cost of different mechanisms of colistin resistance and their impact on virulence in Acinetobacter baumannii. Antimicrob. Agents Chemother. 58, 518-526. doi: 10.1128/AAC.01597-13

Betts, J. W., and Wareham, D. W. (2014). In vitro activity of curcumin in combination with epigallocatechin gallate (EGCG) versus multidrug-resistant Acinetobacter baumannii. BMC Microbiol. 14:172. doi: 10.1186/1471-218014-172

Chabane, Y. N., Marti, S., Rihouey, C., Alexandre, S., Hardouin, J., Lesouhaitier, O., et al. (2014). Characterisation of pellicles formed by Acinetobacter baumannii at the air-liquid interface. PLoS One 9:e111660. doi: 10.1371/journal.pone. 0111660

Clemmer, K. M., Bonomo, R. A., and Rather, P. N. (2011). Genetic analysis of surface motility in Acinetobacter baumannii. Microbiology 157, 2534-2544. doi: 10.1099/mic.0.049791-0

Costerton, J. W., Stewart, P. S., and Greenberg, E. P. (1999). Bacterial biofilms: a common cause of persistent infections. Science 284, 1318-1322. doi: 10.1126/ science. 284.5418 .1318

Cruz-Muniz, M. Y., Lopez-Jacome, L. E., Hernandez-Duran, M., Franco-Cendejas, R., Licona-Limon, P., Ramos-Balderas, J. L., et al. (2017). Repurposing the anticancer drug mitomycin $\mathrm{C}$ for the treatment of persistent Acinetobacter baumannii infections. Int. J. Antimicrob. Agents 49, 88-92. doi: 10.1016/j. ijantimicag.2016.08.022

Darwish Alipour Astaneh, S., Rasooli, I., and Mousavi Gargari, S. L. (2017). Filamentous hemagglutinin adhesin FhaB limits A. baumannii biofilm formation. Front. Biosci. 9, 266-275. doi: 10.2741/e801

Draughn, G. L., Milton, M. E., Feldmann, E. A., Bobay, B. G., Roth, B. M., Olson, A. L., et al. (2018). The structure of the biofilmcontrolling response regulator $\mathrm{BfmR}$ from Acinetobacter baumannii reveals details of its DNA-binding mechanism. J. Mol. Biol. 430, 806-821. doi: 10.1016/j.jmb.2018.02.002

Duarte, S., Gregoire, S., Singh, A. P., Vorsa, N., Schaich, K., Bowen, W. H., et al. (2006). Inhibitory effects of cranberry polyphenols on formation and acidogenicity of Streptococcus mutans biofilms. FEMS Microbiol. Lett. 257, 50-56. doi: 10.1111/j.1574-6968.2006.00147.x

Eijkelkamp, B. A., Stroeher, U. H., Hassan, K. A., Papadimitrious, M. S., Paulsen, I. T., and Brown, M. H. (2011). Adherence and motility characteristics of clinical Acinetobacter baumannii isolates. FEMS Microbiol. Lett. 323, 44-51. doi: 10.1111/j.1574-6968.2011.02362.x

Giles, S. K., Stroeher, U. H., Eijkelkamp, B. A., and Brown, M. H. (2015). Identification of genes essential for pellicle formation in Acinetobacter baumannii. BMC Microbiol. 15:116. doi: 10.1186/s12866-015-0440-6

Howard, A., O'donoghue, M., Feeney, A., and Sleator, R. D. (2012). Acinetobacter baumannii: an emerging opportunistic pathogen. Virulence 3, 243-250. doi: 10.4161/viru. 19700
363 to JL), and by the Priority Research Center Program through the NRF funded by the Ministry of Education (2014R1A6A1031189).

\section{SUPPLEMENTARY MATERIAL}

The Supplementary Material for this article can be found online at: https://www.frontiersin.org/articles/10.3389/fmicb. 2019.00990/full\#supplementary-material

Jaiswal, S., and Mishra, P. (2018). Antimicrobial and antibiofilm activity of curcumin-silver nanoparticles with improved stability and selective toxicity to bacteria over mammalian cells. Med. Microbiol. Immunol. 207, 39-53. doi: 10.1007/s00430-017-0525-y

Kaur, A., Sharma, P., and Capalash, N. (2018). Curcumin alleviates persistence of Acinetobacter baumannii against colistin. Sci. Rep. 8:11029. doi: 10.1038/ s41598-018-29291-z

Kentache, T., Ben Abdelkrim, A., Jouenne, T., Dé, E., and Hardouin, J. (2017). Global dynamic proteome study of a pellicle-forming Acinetobacter baumannii strain. Mol. Cell Proteo. 16, 100-112. doi: 10.1074/mcp.M116.061044

Kostoulias, X., Murray, G. L., Cerqueira, G. M., Kong, J. B., Bantun, F., Mylonakis, E., et al. (2016). Impact of a cross-kingdom signaling molecule of Candida albicans on Acinetobacter baumannii physiology. Antimicrob. Agents Chemother. 60, 161-167. doi: 10.1128/AAC.01540-15

Lee, D., Seo, Y., Khan, M. S., Hwang, J., Jo, Y., Son, J., et al. (2018). Use of nanoscale materials for the effective prevention and extermination of bacterial biofilms. Biotech. Bioprocess Eng. 23, 1-10. doi: 10.1007/s12257-017-0348-0

Lee, J.-H., Park, J. H., Cho, M. H., and Lee, J. (2012). Flavone reduces the production of virulence factors, staphyloxanthin and $\alpha$-hemolysin, in Staphylococcus aureus. Curr. Microbiol. 65, 726-732. doi: 10.1007/s00284-012-0229-x

Lee, J.-H., Regmi, S. C., Kim, J. A., Cho, M. H., Yun, H., Lee, C. S., et al. (2011). Apple flavonoid phloretin inhibits Escherichia coli O157:H7 biofilm formation and ameliorates colon inflammation in rats. Infect. Immun. 79, 4819-4827. doi: 10.1128/IAI.05580-11

Lee, K., Lee, J.-H., Ryu, S. Y., Cho, M. H., and Lee, J. (2014). Stilbenes reduce Staphylococcus aureus hemolysis, biofilm formation, and virulence. Foodborne Pathog. Dis. 11, 710-717. doi: 10.1089/fpd.2014.1758

Liou, M. L., Soo, P. C., Ling, S. R., Kuo, H. Y., Tang, C. Y., and Chang, K. C. (2014). The sensor kinase BfmS mediates virulence in Acinetobacter baumannii. J. Microbiol. Immunol. Infect. 47, 275-281. doi: 10.1016/j.jmii.2012.12.004

Liu, H., Wu, Y. Q., Chen, L. P., Gao, X., Huang, H. N., Qiu, F. L., et al. (2016). Biofilm-related genes: analyses in multi-antibiotic resistant Acinetobacter baumannii isolates from Mainland China. Med. Sci. Monit. 22, 1801-1807. doi: 10.12659/MSM.898959

Luo, L. M., Wu, L. J., Xiao, Y. L., Zhao, D., Chen, Z. X., Kang, M., et al. (2015). Enhancing pili assembly and biofilm formation in Acinetobacter baumannii ATCC19606 using non-native acyl-homoserine lactones. BMC Microbiol. 15:62. doi: 10.1186/s12866-015-0397-5

Manoharan, R. K., Lee, J.-H., Kim, Y.-G., and Lee, J. (2017). Alizarin and chrysazin inhibit biofilm and hyphal formation by Candida albicans. Front. Cell Infect. Microbiol. 7:447. doi: 10.3389/fcimb.2017.00447

Marti, S., Rodriguez-Bano, J., Catel-Ferreira, M., Jouenne, T., Vila, J., Seifert, H., et al. (2011). Biofilm formation at the solid-liquid and air-liquid interfaces by Acinetobacter species. BMC Res. Notes 4:5. doi: 10.1186/1756-0500-4-5

Merken, H. M., and Beecher, G. R. (2000). Liquid chromatographic method for the separation and quantification of prominent flavonoid aglycones. J. Chromatogr. A 897, 177-184. doi: 10.1016/S0021-9673(00)00826-8

Mussi, M. A., Gaddy, J. A., Cabruja, M., Arivett, B. A., Viale, A. M., Rasia, R., et al. (2010). The opportunistic human pathogen Acinetobacter baumannii senses and responds to light. J. Bacteriol. 192, 6336-6345. doi: 10.1128/JB.00917-10

National Committee for Clinical Laboratory Standards (2002). Performance Standards for Antimicrobial Disk and Dilution Susceptibility Tests for Bacteria Isolates From Animals. Approved Standard M31-A2. Wayne, PA: National Committee for Clinical Laboratory Standards. 
Nelson, K. M., Dahlin, J. L., Bisson, J., Graham, J., Pauli, G. F., and Walters, M. A. (2017). The essential medicinal chemistry of curcumin. J. Med. Chem. 60, 1620-1637. doi: 10.1021/acs.jmedchem.6b00975

Pakharukova, N., Tuittila, M., Paavilainen, S., Malmi, H., Parilova, O., Teneberg, S., et al. (2018). Structural basis for Acinetobacter baumannii biofilm formation. Proc. Natl. Acad. Sci. U.S.A. 115, 5558-5563. doi: 10.1073/pnas.1800961115

Paling, F. P., Wolkewitz, M., Bode, L. G. M., Klein Klouwenberg, P. M. C., Ong, D. S. Y., Depuydt, P., et al. (2017a). Staphylococcus aureus colonization at ICU admission as a risk factor for developing $S$. aureus ICU pneumonia. Clin. Microbiol. Infect. 23, 49.e9-49.e14. doi: 10.1016/j.cmi.2016.09.022

Paling, F. P., Wolkewitz, M., Depuydt, P., De Bus, L., Sifakis, F., Bonten, M. J. M., et al. (2017b). P. aeruginosa colonization at ICU admission as a risk factor for developing $P$. aeruginosa ICU pneumonia. Antimicrob. Resist. Infect. Control 6:38. doi: 10.1186/s13756-017-0197-9

Panche, A. N., Diwan, A. D., and Chandra, S. R. (2016). Flavonoids: an overview. J. Nutr. Sci. 5:e47. doi: 10.1017/jns.2016.41

Pour, N. K., Dusane, D. H., Dhakephalkar, P. K., Zamin, F. R., Zinjarde, S. S., and Chopade, B. A. (2011). Biofilm formation by Acinetobacter baumannii strains isolated from urinary tract infection and urinary catheters. FEMS Immunol. Med. Microbiol. 62, 328-338. doi: 10.1111/j.1574-695X.2011.00818.x

Qi, L., Li, H., Zhang, C., Liang, B., Li, J., Wang, L., et al. (2016). Relationship between antibiotic resistance, biofilm formation, and biofilm-specific resistance in Acinetobacter baumannii. Front. Microbiol. 7:483. doi: 10.3389/fmicb.2016. 00483

Rajasekharan, S. K., Ramesh, S., Satish, A. S., and Lee, J. (2017). Antibiofilm and anti- $\beta$-lactamase activities of Burdock root extract and chlorogenic acid against Klebsiella pneumoniae. J. Microbiol. Biotechnol. 27, 542-551. doi: 10.4014/jmb. 1609.09043

Rajsekharan, S. K., Raorane, C. J., and Lee, J. (2018). LED based real-time survival bioassays for nematode research. Sci. Rep. 8:11531. doi: 10.1038/s41598-01830016-5

Runci, F., Bonchi, C., Frangipani, E., Visaggio, D., and Visca, P. (2017). Acinetobacter baumannii biofilm formation in human serum and disruption by gallium. Antimicrob. Agents Chemother. 61:e01563-16. doi: 10.1128/AAC. 01563-16

Russo, T. A., Manohar, A., Beanan, J. M., Olson, R., Macdonald, U., Graham, J., et al. (2016). The response regulator BfmR is a potential drug target for Acinetobacter baumannii. mSphere 1, e82-e16. doi: 10.1128/mSphere.00082-16

Seifert, H., Schulze, A., Baginski, R., and Pulverer, G. (1994). Plasmid DNA fingerprinting of Acinetobacter species other than Acinetobacter baumannii. J. Clin. Microbiol. 32, 82-86.

Singh, A. K., Prakash, P., Singh, R., Nandy, N., Firdaus, Z., Bansal, M., et al. (2017). Curcumin quantum dots mediated degradation of bacterial biofilms. Front. Microbiol. 8:1517. doi: 10.3389/fmicb.2017.01517
Singh, R. (2014). Determination of minimum inhibitory concentration of cycloserine in multidrug- resistant Mycobacterium tuberculosis isolates. Jordan J. Bio. Sci. 7, 139-145. doi: 10.12816/000 8228

Tan, Y., Leonhard, M., Moser, D., Ma, S., and Schneider-Stickler, B. (2018). Antibiofilm efficacy of curcumin in combination with 2aminobenzimidazole against single- and mixed-species biofilms of Candida albicans and Staphylococcus aureus. Colloids Surf. B Biointer. 174, 28-34. doi: 10.1016/j.colsurfb.2018.10.079

Teow, S. Y., Liew, K., Ali, S. A., Khoo, A. S. B., and Peh, S. C. (2016). Antibacterial action of curcumin against Staphylococcus aureus: a brief review. J. Trop. Med. 2016:2853045. doi: 10.1155/2016/2853045

Thompson, R. J., Bobay, B. G., Stowe, S. D., Olson, A. L., Peng, L., $\mathrm{Su}$, Z., et al. (2012). Identification of $\mathrm{BfmR}$, a response regulator involved in biofilm development, as a target for a 2-aminoimidazolebased antibiofilm agent. Biochemistry 51, 9776-9778. doi: 10.1021/bi30 15289

Tomaras, A. P., Dorsey, C. W., Edelmann, R. E., and Actis, L. A. (2003). Attachment to and biofilm formation on abiotic surfaces by Acinetobacter baumannii: involvement of a novel chaperone-usher pili assembly system. Microbiology 149, 3473-3484. doi: 10.1099/mic.0. 26541-0

Tyagi, P., Singh, M., Kumari, H., Kumari, A., and Mukhopadhyay, K. (2015). Bactericidal activity of curcumin I is associated with damaging of bacterial membrane. PLoS One 10:e0121313. doi: 10.1371/journal.pone.0121313

Uppuluri, P., Lin, L., Alqarihi, A., Luo, G., Youssef, E. G., Alkhazraji, S., et al. (2018). The Hyr 1 protein from the fungus Candida albicans is a cross kingdom immunotherapeutic target for Acinetobacter bacterial infection. PLoS Pathog. 14:e1007056. doi: 10.1371/journal.ppat.1007056

Wu, H., Moser, C., Wang, H. Z., Hoiby, N., and Song, Z. J. (2015). Strategies for combating bacterial biofilm infections. Int. J. Oral Sci. 7, 1-7. doi: 10.1038/ijos. 2014.65

Conflict of Interest Statement: The authors declare that the research was conducted in the absence of any commercial or financial relationships that could be construed as a potential conflict of interest.

Copyright (C) 2019 Raorane, Lee, Kim, Rajasekharan, García-Contreras and Lee. This is an open-access article distributed under the terms of the Creative Commons Attribution License (CC BY). The use, distribution or reproduction in other forums is permitted, provided the original author(s) and the copyright owner(s) are credited and that the original publication in this journal is cited, in accordance with accepted academic practice. No use, distribution or reproduction is permitted which does not comply with these terms. 